\title{
Prospective Assessment of the Performance of a New Fine Needle Biopsy Device for EUS-Guided Sampling of Solid Lesions
}

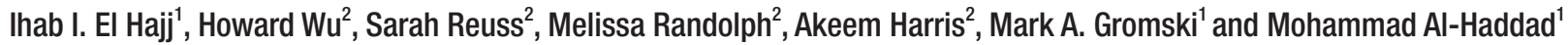 \\ ${ }^{1}$ Division of Gastroenterology, Section of Interventional Endoscopy, ${ }^{2}$ Department of Laboratory Medicine and Cytopathology, Indiana \\ University School of Medicine, Indianapolis, IN, USA
}

Background/Aims: Endoscopic ultrasound-guided fine needle aspiration (EUS-FNA) remains the most common EUS-guided tissue acquisition technique. This study aimed to evaluate the performance of a new Franseen tip fine needle biopsy (FNB) device for EUSguided sampling of solid lesions and compare it with the historical FNA technique.

Methods: Acquire ${ }^{\circledR} 22$ G FNB needle (Boston Scientific Co., Natick, MA, USA) was used for solid tumor sampling (Study group). Tissue was collected for rapid on-site evaluation, and touch and crush preparations were made. Historical EUS-FNA samples obtained using Expect ${ }^{\circledR} 22$ G FNA needle (Boston Scientific Co.) were used as controls (Control group). All specimens were independently evaluated by two cytopathologists blinded to the formal cytopathological diagnosis.

Results: Mean cell block histology scores were significantly higher $(p=0.046)$ in the FNB group (51 samples) despite a significantly lower $(p<0.001)$ mean number of passes compared to the FNA group (50 specimens). The overall diagnostic yields for the FNB vs. FNA groups were $96 \%$ vs. $88 \%$. The degree of tumor differentiation was adequately assessed in all cell block qualifying lesions in the FNB group. Two patients developed post-FNB abdominal pain.

Conclusions: The new Franseen tip FNB device provides histologically superior and cytologically comparable specimens to those obtained by FNA, but with fewer passes. Clin Endosc 2018;51:576-583

Key words: Tissue acquisition; Endoscopic ultrasound-guided fine needle aspiration; Endoscopic ultrasound-guided fine needle biopsy

\section{INTRODUCTION}

Endoscopic ultrasound-guided fine needle aspiration (EUSFNA) remains the most common EUS-guided tissue acquisition technique. However, its reported sensitivity ranges from $65 \%$ to $95 \%{ }^{1,2}$ Several factors can influence the diagnostic yield of any EUS-guided sampling procedure, including the needle type and size, number of passes, lesion-specific charac-

Received: March 10, 2017 Revised: May 21, 2017

Accepted: May 31, 2017

Correspondence: Mohammad Al-Haddad

Division of Gastroenterology, Section of Interventional Endoscopy, Indiana University School of Medicine, 550 University Blvd, Suite 4100, Indianapolis, IN 46202, USA

Tel: +1-317-944-7896, Fax: +1-317-948-8144, E-mail: moalhadd@iu.edu ORCID: https://orcid.org/0000-0003-1641-9976

(cc This is an Open Access article distributed under the terms of the Creative Commons Attribution Non-Commercial License (http://creativecommons.org/ licenses/by-nc/3.0) which permits unrestricted non-commercial use, distribution, and reproduction in any medium, provided the original work is properly cited. teristics, such as location and size, and, finally, endosonographer's experience. ${ }^{1,3,4}$ The presence of rapid on-site cytological evaluation (ROSE) can positively impact the outcome of an EUS-FNA procedure, ${ }^{5}$ although its overall impact on the diagnostic yield of malignant lesions has been recently challenged. ${ }^{6}$ The diagnostic yield of FNA has been consistently high in pancreatic solid lesions, exceeding $85 \%$, ${ }^{7}$ but varies in subepitheial tumors, ranging from $45 \%$ to $90 \%{ }^{8,9}$ A newer generation of fine needle biopsy (FNB) needles became available in the recent years and helped improve the tissue yield compared to the first generation of Trucut biopsy needles. ${ }^{10-12}$ A recent meta-analysis demonstrated comparable diagnostic yield to standard FNA needles with fewer passes. ${ }^{13}$

This study aimed to evaluate the overall diagnostic performance of a new FNB device with a Franseen tip equipped with three cutting edges for EUS sampling of solid lesions (Fig. 1). We sought to assess the cytological and histological yield of the FNB samples compared to those of historical FNA sam- 
ples (controls) obtained using a similar size needle. Our secondary goals were to compare the number of passes required to achieve a cytopathological diagnosis by each method and to assess the cytological quality of smears prepared from FNB samples for immediate cytological interpretation. Finally, we report on the adverse events associated with the use of this new FNB device.

\section{MATERIALS AND METHODS}

\section{Study design and study population}

This single-center study was approved by the Indiana University School of Medicine Institutional Review Board. Consecutive patients referred to a single operator (MAH) for EUS-guided sampling of solid lesions were prospectively enrolled between September 2016 and March 2017. All patients underwent EUS-FNB. ROSE was available in all cases. Patients were excluded if no cell block material was collected or if no standard reference diagnosis was obtainable.

Patient demographics and lesion-related characteristics, including the size and location of target lesions, cytological and pathological findings (in case of lesion surgical resection), and clinical outcomes, were recorded. FNB-related abstracted data included: smear cytology score, cell block histology score, number of passes for smears, number of additional passes for

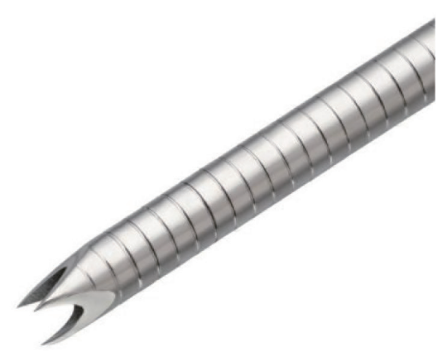

(A)

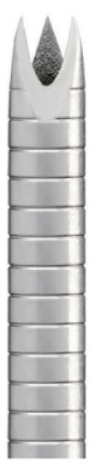

(B)

Fig. 1. Pictures of the new Franseen tip fine needle biopsy (FNB) histology needle. (A) An image of the FNB needle. (B) Closer view of the tip of the FNB needle. cell block, and total number of passes.

EUS-FNA samples previously accrued from solid lesions by the same endosonographer (MAH) between 2013 and 2015 using the Expect ${ }^{\circledR} 22$ G FNA needle (Boston Scientific Co., Natick, MA, USA) were identified and used as controls. ROSE was available in all FNA cases, as this is the standard of care at our center, and was performed in the same way described below for the FNB group.

Matching of controls to cases was performed in two stages: first, consecutive samples obtained from solid lesions were included; subsequently, matching of cases and controls by site (pancreatic vs. non-pancreatic) was attempted. Only controls with available cell block histology score and confirmed diagnosis were included using the same reference diagnostic standard of the Study (FNB) group. All specimens were independently evaluated by two expert cytopathologists (SR and HW) to assess the cytological yield (on smears) and histological yield (on cell block preparations) using a standard scoring system reached by a consensus among our cytopathologists prior to the launching of the study (Table 1, Figs. 2-6). The cytopathologists were blinded to the formal cytopathological diagnosis rendered for clinical care in all FNB cases and controls.

\section{Study definitions}

A clear, non-equivocal diagnosis of malignancy was based on neoplastic cyto-histopathology by endoscopic sampling (FNB or FNA) and/or surgical resection. When cytology was non-diagnostic, the diagnosis of malignancy was considered based on neoplastic EUS impression, clinical course, and imaging findings consistent with malignancy at $\geq 6$ months of follow-up.

\section{EUS-FNB technique and ROSE}

All procedures were performed by the same experienced endosonographer (MAH). EUS-FNB was performed using a linear array echoendoscope (Olympus America Inc., Center Valley, PA, USA) and a 22 G Acquire ${ }^{\circledR}$ FNB needle (Boston Scientific Co.). Monitored anesthesia care sedation was provided by anesthesiologists for all procedures. The slow-pull technique was used to remove the stylet and was followed

Table 1. Cytological and Histological Scoring Systems Used in the Study

\begin{tabular}{|c|c|c|}
\hline Scoring System & Cytology criteria (smears) & Histology criteria (cellblock) \\
\hline Score 1 & $\begin{array}{l}5 \text { or fewer groups ( } \geq 10 \text { cells each group) or }<50 \text { tumor } \\
\text { cells for tumors with dishesive pattern }\end{array}$ & $\begin{array}{l}5 \text { or fewer groups ( } \geq 10 \text { cells each group) or }<50 \text { tumor } \\
\text { cells for tumors with dishesive pattern }\end{array}$ \\
\hline Score 2 & $\begin{array}{l}\text { 6-10 groups ( } \geq 10 \text { cells each group) or } 50-100 \text { tumor cells } \\
\text { for tumors with dishesive pattern }\end{array}$ & $\begin{array}{l}\text { 6-10 groups ( } \geq 10 \text { cells each group) or } 50-100 \text { tumor } \\
\text { cells for tumors with dishesive pattern }\end{array}$ \\
\hline Score 3 & $\begin{array}{l}>10 \text { groups ( } \geq 10 \text { cells each group) or }>100 \text { tumor cells for } \\
\text { tumors with dishesive pattern }\end{array}$ & $\begin{array}{l}>10 \text { groups ( } \geq 10 \text { cells each group) or }>100 \text { tumor cells } \\
\text { for tumors with dishesive pattern }\end{array}$ \\
\hline
\end{tabular}


by application of $5 \mathrm{~mL}$ of negative pressure for 5-10 seconds, during which at least five additional actuations were performed. The fanning technique was used whenever feasible.

Following the needle withdrawal, obtained tissue was processed on site, and touch preparations or crush smears were prepared. Slides were air-dried and stained with modified Wright Giemsa staining for ROSE. If the first smear was negative, the endosonographer performed additional smears until it turned positive, or it was felt that further sampling would not increase the smears yield. All additional visible cores were

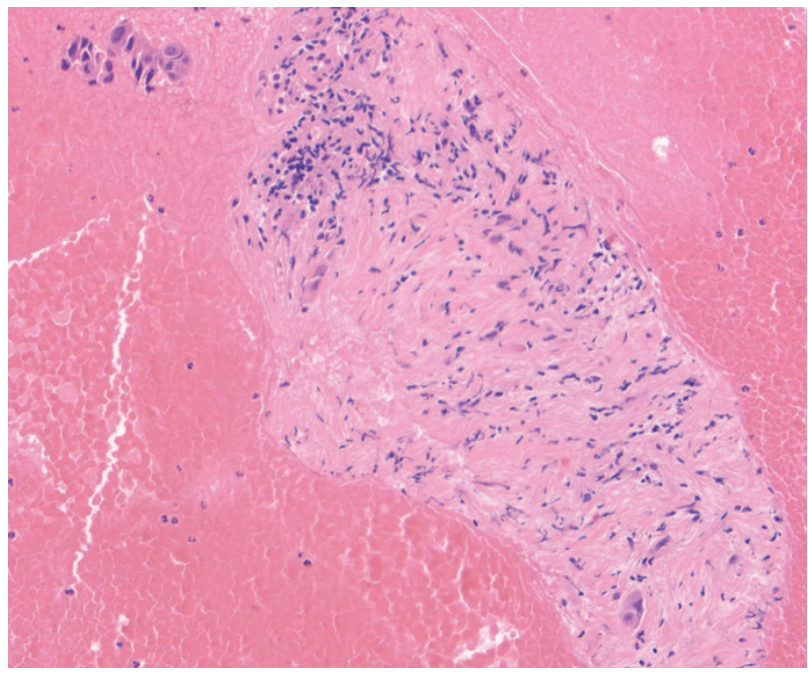

Fig. 2. Fine needle biopsy specimen obtained from a pancreatic head mass. This lesion was sampled using standard fine needle aspiration technique twice prior to referral to our hospital with insufficient cytology. Rare malignant cells seen infiltrating the fibrous stroma. This was assigned a score of 1 on the cell block. The final diagnosis was pancreatic adenocarcinoma (Hematoxylin and eosin, $\times 100$ )

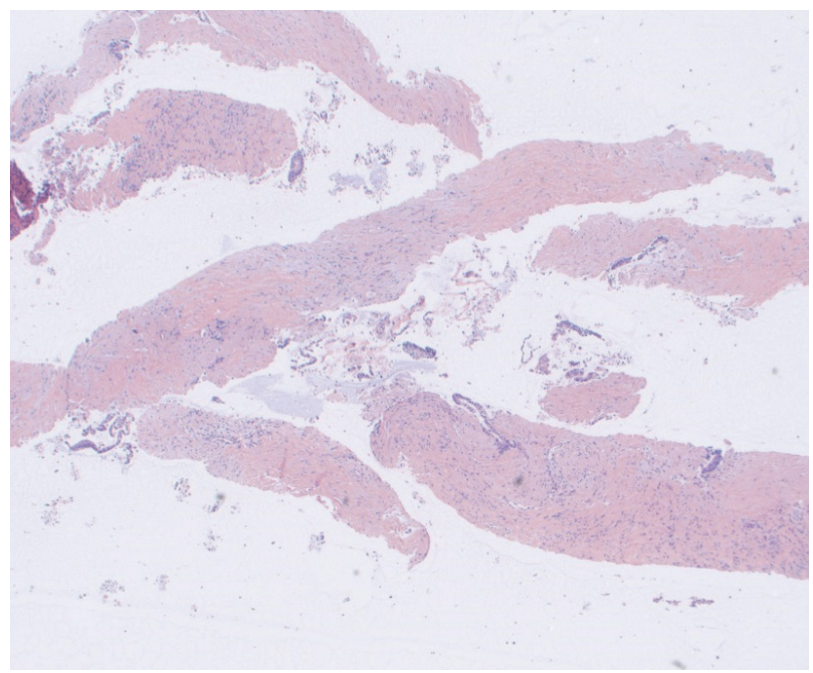

Fig. 3. Fine needle biopsy specimen obtained from a pancreatic body mass This was assigned a score of 2 on cell block. The final diagnosis was pancreatic adenocarcinoma (Hematoxylin and eosin, $\times 20$ ). placed in a cellular preservative (CytoRich Red; Becton Dickinson, Franklin Lakes, NJ, USA) for cell block preparation. Visual inspection of the cell block was performed to ensure that there was sufficient material in terms of the cloudiness of the fluid, and not necessarily relying on the presence of defined whitish cores (some mini-cores can be hard to detect visually and turn the fluid turbid) (Fig. 7).

\section{Follow-up}

Procedure-related outcomes were collected prospectively. Our endoscopy unit routinely telephones all patients within 48-72 hours after the procedures to assess for short-term adverse events. Adverse events were recorded according to the

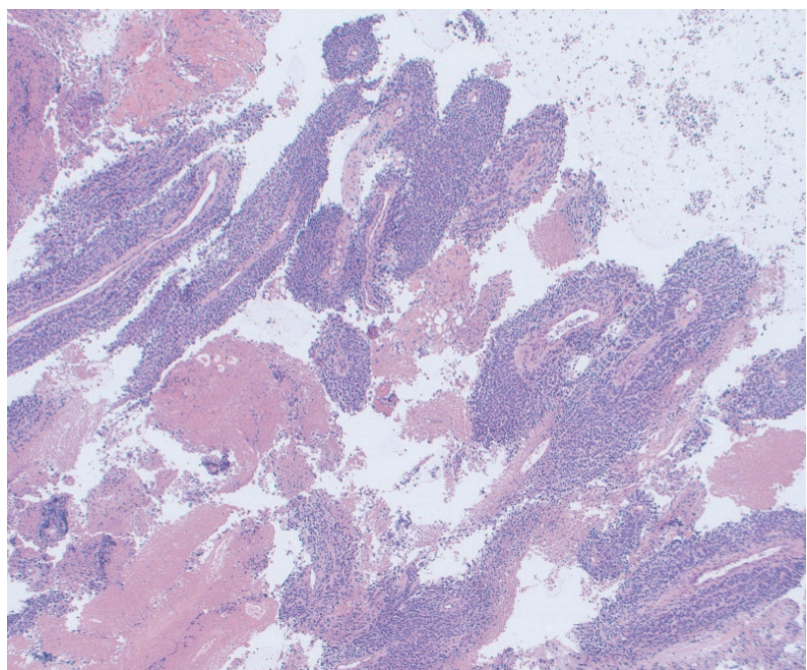

Fig. 4. Fine needle biopsy specimen obtained from a mediastinal mass. This was assigned a score of 3 on cell block. The final diagnosis was poorly differentiated lung cancer (Hematoxylin and eosin, $\times 40$ ).

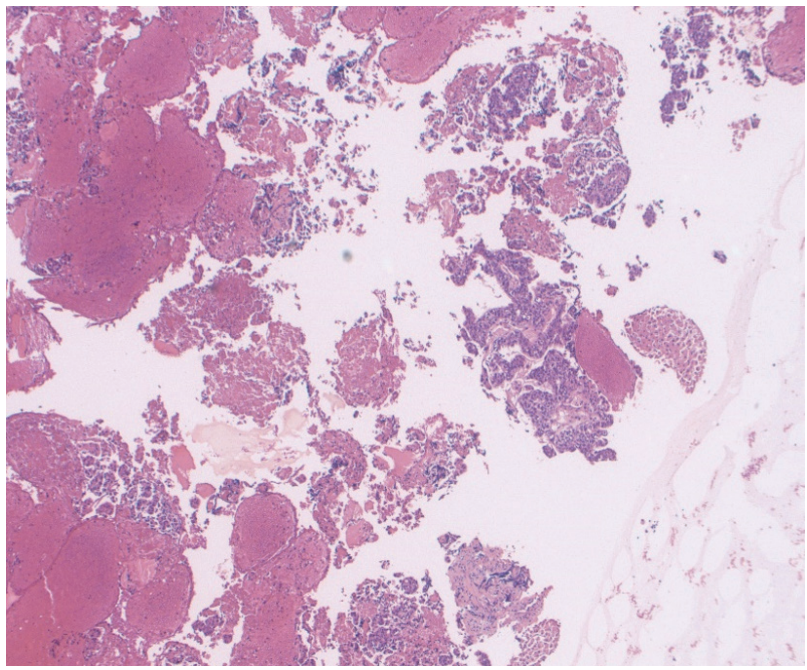

Fig. 5. Fine needle aspiration specimen obtained from a liver mass. This was assigned a score of 3 on cell block. The final diagnosis was metastatic esophageal cancer (Hematoxylin and eosin, $\times 40$ ). 

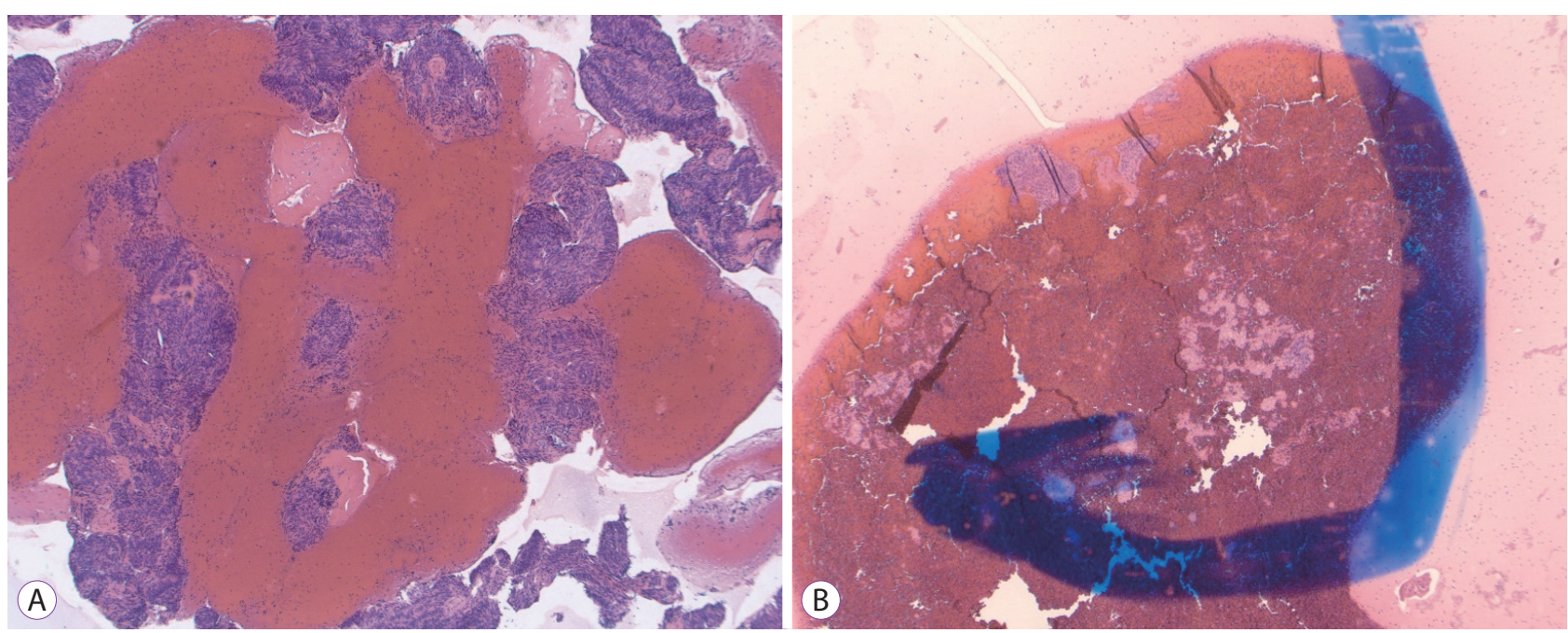

Fig. 6. (A) Fine needle biopsy specimen obtained from a gastric subepithelial mass. Extensive bands of spindled cells with high cellularity are noted in this cell block. The final diagnosis of gastrointestinal stromal tumor was confirmed on c-Kit staining (Hematoxylin and eosin $[H \& E], \times 40)$. (B) Fine needle aspiration specimen pulled from the archives from a gastric subepithelial mass of similar morphology and dimensions to the one presented in (A). Although this preparation is still diagnostic for gastrointestinal stromal tumor, substantially fewer groups of spindled cells are noted in this cell block (H\&E, $\times 40$ ).

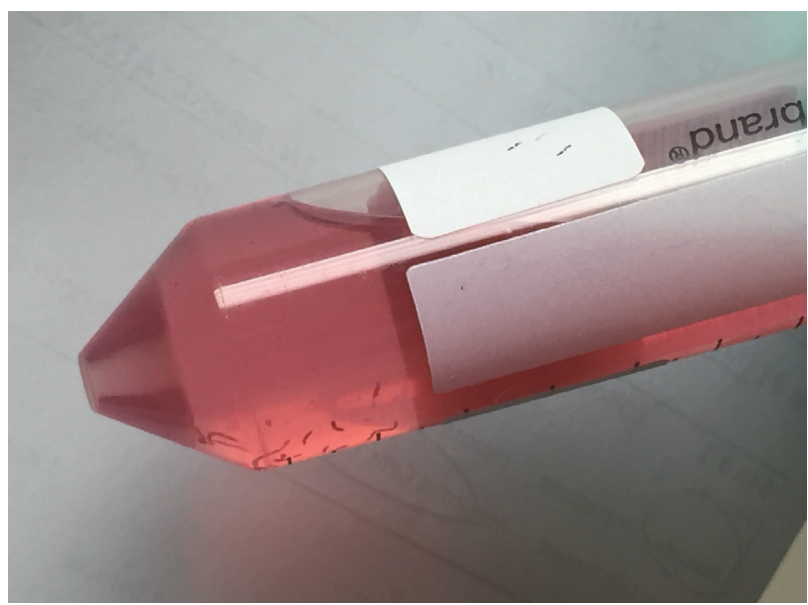

Fig. 7. As opposed to the blood-tinged preservative solution with a clot on the bottom of the tube seen with standard fine needle aspiration cell blocks, fine needle biopsy blocks tend to be less bloody with several visible tan-colored tissue fragments (cores).

published American Society of Gastrointestinal Endoscopy criteria. ${ }^{14}$ Additional follow-up information after EUS was performed by review of medical charts and contact with the referring physicians (if necessary).

\section{Data analysis}

Continuous variables were reported as mean \pm standard deviation. Categorical variables were presented as numbers and percentages. Sensitivity, specificity, positive predictive value, negative predictive value, and accuracy were calculated. To determine the differences between the groups, the Student's $t$-test was used for continuous variables and chi-squared or Fisher's exact test was used for categorical variables. The sample size was not estimated a priori as this is the first study conducted with this novel needle. We chose 50 samples in the test group and a similar number of controls as a convenience sample. Two-sided $p$-values $<0.05$ were considered statistically significant. Statistical analysis was performed using SPSS 21.0 software for Windows (SPSS Inc., Chicago, IL, USA).

\section{RESULTS}

Fifty patients (28 males) underwent EUS-FNB for 51 solid lesions between September 2016 and March 2017 at Indiana University Hospital and comprised the Study group. Lesion and sampling results characteristics are summarized in Table 2. A neoplastic diagnosis was confirmed in the vast majority of patients (48/50; 96\%) based on cytology. Resection surgical pathology confirmed the cytological diagnosis in 12 patients (24\%).

Fifty FNA specimens from solid lesions were pulled from our cytology archives and were independently reviewed (Control group). Mean cell block histology scores were significantly higher in the FNB group $(p=0.046)$ despite an overall lower mean number of passes compared to the FNA group $(p<0.001$, Table 2). The overall diagnostic yield of FNB was $96 \%$ compared to $88 \%$ for the FNA group, but the difference did not reach statistical significance.

The degree of tumor differentiation for primary tumors, origin of metastatic lesions, and mitotic index in neuroendocrine and stromal tumors $(n=18)$ was adequately assessed in 
all lesions based on cell block studies in the FNB group (Table 3). The performance characteristics (sensitivity, specificity, and accuracy) of the two sampling methods assessed in this study (FNB vs. FNA) are summarized in Table 4.

\section{Adverse events}

All adverse events were classified as mild, and were recognized during or immediately after the procedure. ${ }^{14}$

Two patients (4\%) developed post-procedural abdominal

Table 2. Lesion and Sampling Results Characteristics of the Fine Needle Biopsy and Fine Needle Aspiration Cohorts

\begin{tabular}{lccc}
\hline Lesion and sampling characteristics & FNB $(\boldsymbol{n = 5 1 )}$ & FNA $(\boldsymbol{n = 5 0 )}$ & p-value \\
\hline Mean size (maximal dimension, $\mathrm{cm})$ & 2.98 & 2.90 & NS \\
Location: Pancreatic $(n)$ & 23 & 20 & NS \\
Non pancreatic $(n)$ & 28 & 30 & NS \\
Cytology scores of smears (mean) & 2.75 & 2.86 & 0.056 \\
Number of passes for smears (mean) & 2.07 & 2.44 & 0.046 \\
Cell block histology score (mean) & 2.32 & 2.02 & $<0.001$ \\
Number of additional passes for cell block (mean) & 0.74 & 1.68 & $<0.001$ \\
Total number of passes (mean) & 2.88 & 3.82 & NS \\
Overall diagnostic yield & $96 \%$ & $88 \%$ & \\
\hline
\end{tabular}

FNB, fine needle biopsy; FNA, fine needle aspiration; NS, non significant.

Table 3. Confirmed Clinical Outcomes of All 50 Patients Included in the Study

\begin{tabular}{|c|c|}
\hline Site of FNB $(n=51)^{\mathrm{a})}$ & Final reference diagnosis $\left.(n=50)^{\mathrm{a}}\right)$ \\
\hline $\begin{array}{l}\text { Pancreas }(n=21)^{\mathrm{b})} \\
\text { Head and Uncinate }(n=11) \\
\text { Body }(n=5) \\
\text { Tail }(n=5)\end{array}$ & $\begin{array}{l}\text { Adenocarcinoma }(n=9) ; \text { Chronic pancreatitis }(n=1) \text {; NET }(n=1) \\
\text { Adenocarcinoma }(n=4) ; \text { Chronic pancreatitis }(n=1) ; \\
\text { Adenocarcinoma }(n=4) ; \text { Well differentiated NET }(n=1)\end{array}$ \\
\hline $\begin{array}{l}\text { SET }(n=8) \\
\text { Esophageal }(n=3) \\
\text { Gastric }(n=4) \\
\text { Duodenum }(n=1)\end{array}$ & $\begin{array}{l}\text { Leiomyoma }(n=3) \\
\text { GIST }(n=4) \\
\text { GIST }(n=1)\end{array}$ \\
\hline Lymph nodes $(n=6)$ & $\begin{array}{l}\text { Metastatic adenocarcinoma }(n=4) ; \text { Large B cell lymphoma }(n=1) \\
\text { Metastatic melanoma }(n=1)\end{array}$ \\
\hline Liver lesions $(n=5)$ & Metastatic adenocarcinoma $(n=3)$; Metastatic NET $(n=1)$; Metastatic melanoma $(n=1)$ \\
\hline Distal bile duct masses $(n=3)$ & Cholangiocarcinoma $(n=3)$ \\
\hline Mediastinal masses $(n=2)$ & Poorly differentiated carcinoma $(n=1)$; Squamous cell carcinoma $(n=1)$ \\
\hline Retroperitoneal masses $(n=2)$ & Local recurrence of previously resected pancreatic adenocarcinoma $(n=2)$ \\
\hline $\begin{array}{l}\text { Miscellaneous }(n=4) \\
\text { Gastric wall thickening }(n=1) \\
\text { Perirectal mass }(n=1) \\
\text { Left adrenal mass }(n=1) \\
\text { Pelvic mass }(n=1)\end{array}$ & $\begin{array}{l}\text { Adenocarcinoma (linitis plastica) } \\
\text { Local recurrence of previously resected rectal adenocarcinoma } \\
\text { Metastatic lung adenocarcinoma } \\
\text { Teratoma }\end{array}$ \\
\hline
\end{tabular}

FNB, fine needle biopsy; NET, neuroendocrine tumor; SET, subepitheial tumor; GIST, gastrointestinal stromal tumor.

${ }^{a}$ A single patient with metastatic melanoma underwent sampling of liver lesion and a lymph node, both were metastatic.

${ }^{b}$ Four patients diagnosed eventually with adenocarcinoma based on FNB had previous insufficient fine needle aspiration sampling at other institutions.

Table 4. Performance Characteristics of the 2 Sampling Methods Assessed in This Study (FNB vs. FNA)

\begin{tabular}{lccc}
\hline & Sensitivity (95\% CI) & Specificity (95\% CI) & Accuracy \\
\hline FNB group & $95.9 \%(86.02 \%$ to $99.50 \%)$ & $100 \%(15.81 \%$ to $100.00 \%)$ & $96 \%$ \\
FNA group & $87.23 \%(74.26 \%$ to $95.17 \%)$ & $100 \%(29.24 \%$ to $100.00 \%)$ & $88 \%$ \\
\hline
\end{tabular}

FNB, fine needle biopsy; FNA, fine needle aspiration; CI, confidence interval. 


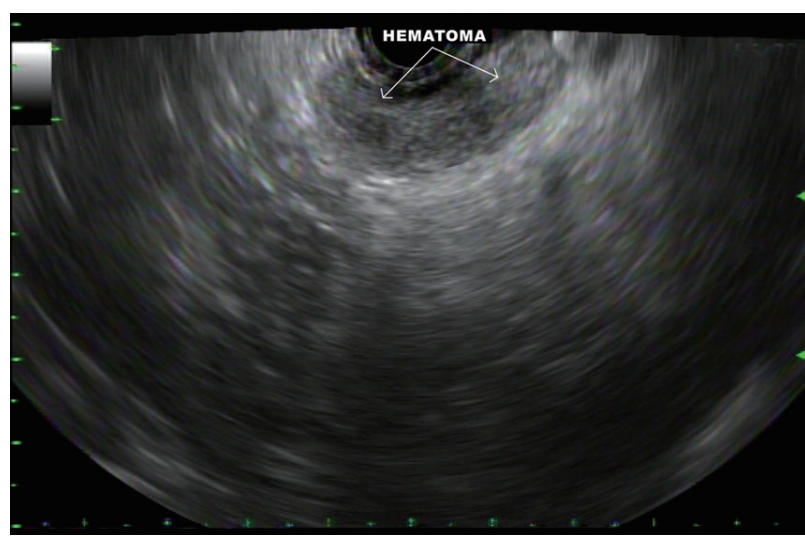

Fig. 8. A hematoma that developed between the gastric wall and the pancreatic neck after fine needle biopsy of a pancreatic body mass.

pain after pancreas mass FNB (confirmed adenocarcinoma on cytology). This required extended observation in the recovery room for 3 hours in one patient and 23-hour admission to the hospital for observation and pain management in another patient.

In two cases, a hematoma developed outside the gastric and duodenal walls during FNB of the pancreatic body (Fig. 8) and head masses, respectively. Both patients underwent 4 passes each. The hematomas were monitored for 10 minutes and appeared stable on EUS. This did not result in clinically apparent adverse outcomes and both patients were discharged home after 90 minutes of observation without the need for further interventions.

No other adverse event was reported during or immediately after the procedure and up to 72 hours of follow-up.

\section{DISCUSSION}

EUS-FNB is increasingly performed to sample solid lesions, but is currently reserved to cases where FNA cytology fails or when tissue architecture is essential for the diagnosis. ${ }^{1}$ The performance of earlier Trucut core biopsy devices (QuickCore; Cook Medical, Limerick, Ireland) was suboptimal due to mechanical failures of its spring loading mechanism. ${ }^{15}$ A newer needle with unique reversed bevel design was released in 2011 (ProCore; Cook Medical) and remains the most widely studied needle. ${ }^{13}$ Superior histologic yield has been demonstrated in one recent randomized study comparing the ProCore to the QuickCore needles. ${ }^{12}$ However, multiple studies failed to demonstrate the superiority of this needle for sampling adequacy or diagnostic accuracy over conventional EUS-FNA needles. ${ }^{16} \mathrm{~A}$ recent meta-analysis of nine studies including 576 patients demonstrated no significant difference in diagnostic adequacy ( $75 \%$ vs. $89 \%)$, diagnostic accuracy ( $85 \%$ vs. $86 \%)$, or rate of histological core specimen acquisition (78\% vs. $77 \%$ ) between the ProCore and standard FNA needles. ${ }^{13}$ More recently, the performance of the ProCore needle was compared to that of a newer core biopsy device with a novel forktip with opposing bevel design and multiple cutting surfaces $\left(\right.$ Sharkcore $^{\mathrm{TM}}$; Medtronic, Minneapolis, MN, USA). ${ }^{17}$ In this study, the opposing bevel needle provided significantly higher sensitivity (71.1\% vs. $90.1 \%)$ and overall accuracy ( $74 \%$ vs. $92 \%$ ) for discriminating malignant from benign solid pancreatic masses.

Our study aimed to assess another new core biopsy device with three cutting surfaces and a Franseen shaped tip. We demonstrated that this device provides a histologically superior sample with fewer passes compared to conventional FNA needles of the same size. The superiority of FNB samples have been recently reported with the fork-tip device. ${ }^{18,19}$ The current study agrees with the results of those two studies from two perspectives: first, the novel needle tip design appears to be a significant factor enhancing tissue acquisition; second, this same tip design could carry a slightly higher risk for tissue trauma, resulting in bleeding and post-procedural pain. In our series, as opposed to the results reported by Rodrigues-Pinto et al., ${ }^{19}$ bleeding was self-limited in all cases with no further interventions were needed.

We have also demonstrated that the smears prepared from such needles do not appear to be inferior to FNA smears for rapid review purposes. This is likely due to the rather fragmented nature of the cores accrued, allowing sheets of cells to smear in thin layers to allow cytological interpretation whether on alcohol fixed or on-site dipped smears.

A recent preliminary descriptive report on this needle demonstrated that diagnostic material for ROSE and histology could be obtained in $97 \%$ of patients..$^{20}$ Our study demonstrated a higher diagnostic accuracy in the FNB group compared to FNA group, although the difference did not reach statistical significance. This may be related to the fact that we applied strict cytological criteria to assess smears and cell block specimens which relied on manual counting of the smears and cell blocks. Since the majority of sampled lesions were solid pancreatic masses, we drew the conclusions that in such cases, three passes are sufficient for establishing the diagnosis with over $90 \%$ diagnostic adequacy.

The yield of EUS-guided tissue acquisition is influenced by many factors. ${ }^{1,3,4}$ In the current study, we attempted to standardize many of the variables known to impact the outcomes of sampling. For example, a standard FNB sampling technique was performed by the same endosonographer, using the same needle gauge and the same standard cytological processing techniques. In addition, the vast majority of lesions in our FNB cohort were unequivocally neoplastic, and the few with 
negative cytology had sufficient follow-up to rule out a malignant outcome. Finally, our cytopathology team has devised a scoring system that minimizes subjectivity in assessing cellularity of smears and cell block material. This system relies on a manual count of the number of diagnostic cells (most commonly tumors) and not on the presence of any nucleated cell. Similar scoring systems have been developed with variable cell count brackets. ${ }^{21}$

The diagnostic yield of FNA in pancreatic masses remains high, with a recent meta-analysis showing an EUS-FNA pooled sensitivity and specificity for diagnosing solid pancreatic neoplasms of $85 \%$ and $98 \%$, respectively. ${ }^{22}$ Despite that, a recent cost-effectiveness analysis from the perspective of a third-party payer showed that FNB was more cost-effective than FNA in sampling both pancreatic and non-pancreatic masses. ${ }^{23}$ Our study demonstrates the utility of routine use of a core biopsy device for solid lesion sampling during EUS.

In addition, this technique appeared to be safe. All adverse events were classified as mild, and were recognized during or immediately after the procedure. Two patients developed post-procedural abdominal pain, and two patients developed hematoma outside the gastric and duodenal walls. No other adverse events were reported during or immediately after the procedure and up to 72 hours of follow-up.

We recognize several limitations to our study. This was not a prospective randomized controlled study. Different solid lesions from different organs were prospectively sampled using the FNB needle only; thus, a direct comparison of the performance of FNB to FNA needles for the same lesion(s) could not be assessed. An important part of the assessment of this new tissue acquisition device was to examine its performance for the purposes of ROSE. As has been endorsed by several studies and key opinion leaders, we continue to rely heavily on ROSE and hence we describe the utility of FNB devices in the context of ROSE. The natural next step is to assess its performance without ROSE, but this study was an early clinical utility study. In our cohort we did not have sufficient number of patients to comment on the performance of this device in specific situations like lymphoma, gastrointestinal stromal tumor, or autoimmune pancreatitis. Finally, we have chosen smears and cell block technique instead of histological exam (typically submitted in formalin). Moreover, our sample size was relatively small, since this is the first study to assess this specific needle platform in a controlled fashion. This could have been underpowered to detect true differences between the FNB and FNA groups in diagnostic accuracy. Despite that, several endpoints, including the total number of passes and the number of passes dedicated to cell block, met the statistical significance. Finally, we confirmed the results of previous reports showing that FNB devices are overall associated with fewer number of passes. ${ }^{13}$ Additionally, we acknowledge that there may be a learning curve to using a new needle platform that was "mastered" during the span of the study.

In conclusion, the new Franseen tip biopsy needle enables sufficient acquisition of histological-quality samples under EUS-guidance. Due to the availability of various sizes and the overall safety of such devices, an FNB exclusive approach to sampling all solid lesions appears feasible. Further studies are needed to characterize the performance of this device in lesions of various origins and locations.

Note

An abstract of this study was accepted for poster presentation at the Digestive Diseases Week, 2017, Washington, DC and at 2017 American College of Gastroenterology Annual Meeting in Orlando, FL, USA.

Conflicts of Interest

The authors have no financial conflicts of interest.

\section{REFERENCES}

1. Wani S, Muthusamy VR, Komanduri S. EUS-guided tissue acquisition: an evidence-based approach (with videos). Gastrointest Endosc 2014;80:939-959.e7.

2. Dumonceau JM, Deprez PH, Jenssen C, et al. Indications, results, and clinical impact of endoscopic ultrasound (EUS)-guided sampling in gastroenterology: European Society of Gastrointestinal Endoscopy (ESGE) clinical guideline - updated January 2017. Endoscopy 2017;49:695-714.

3. Mohamadnejad M, Mullady D, Early DS, et al. Increasing number of passes beyond 4 does not increase sensitivity of detection of pancreatic malignancy by endoscopic ultrasound-guided fine-needle aspiration. Clin Gastroenterol Hepatol 2017;15:1071-1078.e2.

4. Rong L, Kida M, Yamauchi H, et al. Factors affecting the diagnostic accuracy of endoscopic ultrasonography-guided fine-needle aspiration (EUS-FNA) for upper gastrointestinal submucosal or extraluminal solid mass lesions. Dig Endosc 2012;24:358-363.

5. Shield PW, Cosier J, Ellerby G, Gartrell M, Papadimos D. Rapid on-site evaluation of fine needle aspiration specimens by cytology scientists: a review of 3032 specimens. Cytopathology 2014;25:322-329.

6. Wani S, Mullady D, Early DS, et al. The clinical impact of immediate on-site cytopathology evaluation during endoscopic ultrasound-guided fine needle aspiration of pancreatic masses: a prospective multicenter randomized controlled trial. Am J Gastroenterol 2015;110:1429-1439.

7. Savides TJ, Donohue M, Hunt G, et al. EUS-guided FNA diagnostic yield of malignancy in solid pancreatic masses: a benchmark for quality performance measurement. Gastrointest Endosc 2007;66:277-282.

8. Sepe PS, Moparty B, Pitman MB, Saltzman JR, Brugge WR. EUS-guided FNA for the diagnosis of GI stromal cell tumors: sensitivity and cytologic yield. Gastrointest Endosc 2009;70:254-261.

9. Mekky MA, Yamao K, Sawaki A, et al. Diagnostic utility of EUS-guided FNA in patients with gastric submucosal tumors. Gastrointest Endosc 2010;71:913-919.

10. Varadarajulu S, Fraig M, Schmulewitz N, et al. Comparison of EUS-guided 19-gauge trucut needle biopsy with EUS-guided fine-needle aspiration. Endoscopy 2004;36:397-401.

11. Levy MJ, Jondal ML, Clain J, Wiersema MJ. Preliminary experience with an EUS-guided trucut biopsy needle compared with EUS-guided FNA. Gastrointest Endosc 2003;57:101-106. 
12. DeWitt J, Cho CM, Lin J, et al. Comparison of EUS-guided tissue acquisition using two different 19-gauge core biopsy needles: a multicenter, prospective, randomized, and blinded study. Endosc Int Open 2015;3:E471-E478.

13. Bang JY, Hawes R, Varadarajulu S. A meta-analysis comparing ProCore and standard fine-needle aspiration needles for endoscopic ultrasound-guided tissue acquisition. Endoscopy 2016;48:339-349.

14. Cotton PB, Eisen GM, Aabakken L, et al. A lexicon for endoscopic adverse events: report of an ASGE workshop. Gastrointest Endosc 2010;71:446-454

15. Bang JY, Varadarajulu S. Procore and flexible 19 gauge needle can replace trucut biopsy needle? Clin Endosc 2013;46:503-505.

16. Witt BL, Adler DG, Hilden K, Layfield LJ. A comparative needle study: EUS-FNA procedures using the HD ProCore ${ }^{\mathrm{TM}}$ and EchoTip ${ }^{\circledR}$ 22-gauge needle types. Diagn Cytopathol 2013;41:1069-1074.

17. Nayar MK, Paranandi B, Dawwas MF, et al. Comparison of the diagnostic performance of 2 core biopsy needles for EUS-guided tissue acquisition from solid pancreatic lesions. Gastrointest Endosc 2017;85:10171024.

18. Kandel P, Tranesh G, Nassar A, et al. EUS-guided fine needle biopsy sampling using a novel fork-tip needle: a case-control study. Gastrointest Endosc 2016;84:1034-1039.

19. Rodrigues-Pinto E, Jalaj S, Grimm IS, Baron TH. Impact of EUS-guided fine-needle biopsy sampling with a new core needle on the need for onsite cytopathologic assessment: a preliminary study. Gastrointest Endosc 2016;84:1040-1046.

20. Bang JY, Hebert-Magee S, Hasan MK, Navaneethan U, Hawes R, Varadarajulu S. Endoscopic ultrasonography-guided biopsy using a Franseen needle design: initial assessment. Dig Endosc 2017;29:338-346.

21. Mounzer R, Yen R, Marshall C, et al. Interobserver agreement among cytopathologists in the evaluation of pancreatic endoscopic ultrasound-guided fine needle aspiration cytology specimens. Endosc Int Open 2016;4:E812-E819.

22. Hewitt MJ, McPhail MJ, Possamai L, Dhar A, Vlavianos P, Monahan KJ. EUS-guided FNA for diagnosis of solid pancreatic neoplasms: a meta-analysis. Gastrointest Endosc 2012;75:319-331.

23. Aadam AA, Wani S, Amick A, et al. A randomized controlled cross-over trial and cost analysis comparing endoscopic ultrasound fine needle aspiration and fine needle biopsy. Endosc Int Open 2016;4:E497-E505. 